\title{
Asociación de los trastornos externalizados y la edad de inicio en pacientes con trastorno bipolar tipo I y II \\ ¿Son los síntomas de los trastornos externalizados predictores de una edad de inicio más temprano?
}

\author{
Lino Palacios-Cruz, ${ }^{1}$ Adriana Arias-Caballero, ${ }^{1}$ Francisco Cortés Sotres, ${ }^{2}$ Francisco de la Peña-Olvera, ${ }^{3}$ \\ Miriam Feria Aranda, ${ }^{1}$ Marcela Cárdenas Godinez, ${ }^{1}$ Rogelio Apiquian-Guitart, ${ }^{4}$ \\ Alfonso Cabrera-Lagunes, ${ }^{5}$ Carlos Berlanga, ${ }^{6}$ Ana Fresán, ${ }^{6}$ Gerhard Heinze-Martin ${ }^{7}$
}

Artículo original

\begin{abstract}
SUMMARY
Background

Early onset mania (MIMT), compared with adolescent onset mania (MIA), has a different clinical presentation of classic mania adult onset (MIEA). Patients with MIA have a course more like the MIEA. Externalizing disorders (ADHD, ODD, and TC) have been associated with an earlier age of onset of bipolar disorder and as a marker of poor prognosis. Our goal is to determine the frequency of symptoms related to disruptive behavior disorders in patients with bipolar disorder start very early, early and adult evaluated retrospectively.

\section{Methods}

The total sample $(\mathrm{N}=64)$ of adolescent and adult patients was obtained from the National Institute of Psychiatry (INPRF) clinics. The diagnosis was confirmed by the research team. Patients signed the informed asentment and consentment. We applied the K SADS PL Mexico, MINI and MINI KID. We used the EEPE-AA for externalizing disorders.
\end{abstract}

\section{Results}

There were significant differences in scores compared by EEPE AA EIED groups in the Inattention subscale for GIMT. The presence of $A D H D, O D D, T C$ and Suicide Risk in the time of evaluation was significantly associated with an earlier onset.

\section{Discussion and conclusion}

Our data supports the clinical utility and importance of separating the TBP by age of onset. The detection of externalizing disorders may speak of an early onset of the disorder and may also have implications for prognosis and psychopharmacological treatment, since the TBP- onset childhood to adulthood remains similar and difficult to manage. This suggests that we must have a longitudinal view of this disorder.

Key words: Externalizing disorders, bipolar disorder, age of onset.

\section{RESUMEN}

\section{Antecedentes}

La manía de inicio muy temprano (MIMT), comparada con la manía de inicio en la adolescencia (MIA), tiene una presentación clínica distinta a la manía clásica de inicio en la adultez (MIEA). Los pacientes con MIA tienen un curso más parecido a la MIEA. Los trastornos externalizados (TDAH, TND y TC) se han asociado con una edad de inicio más temprano del TBP y como un marcador de mal pronóstico. Nuestro objetivo es determinar la frecuencia de los síntomas relacionados con los trastornos de la conducta disruptiva en pacientes con TBP de inicio muy temprano, temprano $y$ en el adulto evaluados de manera retrospectiva.

\section{Método}

La muestra total $(\mathrm{N}=64)$ de pacientes adolescentes y adultos se obtuvo de distintas clínicas del Instituto Nacional de Psiquiatría (INPRF). El diagnóstico fue confirmado por el equipo de investigación y se solicitó la firma del asentimiento y consentimiento informado. Se aplicaron el K SADS PL México, MINI y MINI KID. Se utilizó la EEPE-AA para los trastornos externalizados.

\section{Resultados}

Se encontraron diferencias significativas en las puntuaciones del EEPE AA comparadas por los grupos de EIED, en la Subescala de Inaten-

\footnotetext{
Programa Detección Oportuna y Estudio del TDAH a lo largo de la vida, PROMETEO/Subdirección de Investigaciones Clínicas, Instituto Nacional de Psiquatría Ramón de la Fuente Muñiz.

Departamento de Apoyo Académico a los alumnos de posgrado, Instituto Nacional de Psiquiatría Ramón de la Fuente Muñiz.

Departamento de Fomento a la Investigación, Instituto Nacional de Psiquiatría Ramón de la Fuente Muñiz.

ARETE Proyectos y Administración S.C., México.

Facultad de Medicina. Universidad Veracruzana, Xalapa, Ver. México.

Subdirección de Investigaciones Clinicas, Instituto Nacional de Psiquiatría Ramón de la Fuente Muñiz.

Departamento de Psiquiatría y Salud Mental, Universidad Nacional Autónoma de México.

Correspondencia: Dr. Lino Palacios. Programa Detección Oportuna y Estudio del TDAH a lo largo de la vida, PROMETEO/Subdirección de Investigaciones Clínicas, Instituto Nacional de Psiquatría Ramón de la Fuente Muñiz. Calz. México-Xochimilco 101, San Lorenzo Huipulco, Tlalpan, 14370 México, DF. E-mail: palacioslino@gmail.com
} 
ción para el GIMT. La presencia de TDAH, TND, TC y riesgo suicida en el momento de la evaluación se relacionó significativamente con un inicio más temprano.

\section{Discusión y conclusión}

Nuestros datos apoyan la importancia y utilidad clínica de separar el TBP por edad de inicio y la detección de trastornos externalizados podría hablarnos de un inicio temprano del trastorno. Asimismo, tiene implicaciones en el pronóstico y tratamiento psicofarmacológico, ya que el TBP de inicio en la infancia permanece en la adultez con características similares, así como de difícil manejo. Es necesario tener una visión longitudinal de este padecimiento.

Palabras clave: Trastornos externalizados, trastorno bipolar, edad de inicio.

\section{ANTECEDENTES}

El trastorno bipolar (TBP) es una enfermedad que se manifiesta entre el 1 y $2 \%$ de la población general adulta mexicana. ${ }^{1,2}$ La mayoría de los clínicos e investigadores concuerdan en que el diagnóstico de TBP es fenomenológicamente heterogéneo y probablemente representa varios subtipos distintos. $^{3}$

El estudio del TBP a partir de la edad de inicio es un abordaje útil para la explicación de la heterogeneidad de la presentación clínica entre pacientes. ${ }^{4,5}$ Actualmente se acepta que la manía de inicio muy temprano (MIMT, anterior a los 13 años de edad), comparada con la manía de inicio en la adolescencia (MIA, entre los 13 y los 18 años de edad), tiene una presentación clínica distinta a la manía clásica que frecuentemente se inicia en la edad adulta (MIEA), ${ }^{6}$ es decir, con más síntomas psicóticos que incluyen los síntomas de primer rango. ${ }^{7}$ Por lo antes descrito, es común que los pacientes con MIMT sean mal diagnosticados como esquizofrénicos ${ }^{7,8}$ o como portadores de un trastorno esquizoafectivo. ${ }^{9}$

Los pacientes con manía de inicio en la adolescencia tienen un curso más parecido a la manía clásica de inicio en la edad adulta, ${ }^{10,11} \mathrm{y}$ aun cuando los pacientes con un inicio más temprano presentaron mayor probabilidad de recibir tratamiento a largo plazo, más síntomas psicóticos y mayor cronicidad, comparados con los pacientes con inicio en la edad adulta, el pronóstico a 15 años en ambos grupos fue similar. ${ }^{6}$

Recientemente se ha sugerido la existencia de un subtipo de TBP desde la perspectiva del desarrollo que puede ser identificado a partir de la edad de inicio. ${ }^{10,12-18}$ Algunos de estos autores han mencionado que el TBP de inicio muy temprano (antes de los 13 años) y de inicio en la adolescencia se asocian con un curso más grave, con la presencia de trastornos de ansiedad, una mayor comorbilidad con trastornos externalizados, consumo de sustancias, ciclados rápidos y mayores tasas de conducta suicida en comparación con el TBP de inicio en la vida adulta, lo que sugiriere que una edad de inicio temprana o muy temprana predice un curso más complejo y grave del TBP..$^{10,17}$

En relación con la comorbilidad con los trastornos externalizados, es decir el trastorno por déficit de atención con hiperactividad (TDAH), el trastorno negativista desafiante (TND) y el trastorno de conducta (TC), se ha mostrado que del 57 al 97\% de los niños con manía cumplen con criterios para TDAH. ${ }^{19-21}$ Faraone et al. encontraron que los adolescentes con manía de inicio en la infancia tenían los mismos índices de TDAH comórbido que los niños maniacos (90\%) y que ambos grupos tenían índices de TDAH comórbido más altos que los adolescentes con manía de inicio en la adolescencia (60\%). ${ }^{22}$ En el caso del TC, los mismos autores encontraron que el $69 \%$ de los niños y adolescentes bipolares presentaban esta comorbilidad. La presencia de trastornos externalizados, principalmente TDAH, se ha señalado como un marcador de mal pronóstico ya que indica una edad de inicio más temprana, confirmado tanto en estudios

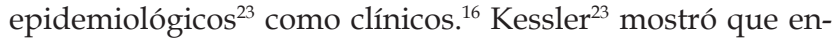
tre aquellos con TDAH existió una probabilidad 7.4 veces mayor (IC al 95\% 4.6 a 12) de presentar un TBP comparado con aquellos que no tenían TDAH. Por otra parte, el 21.2 de los adultos con TBP presentaron TDAH comparado sólo con el 3.5\% de aquellos sin TBP.

Ante esta evidencia, el presente estudio tuvo el objetivo principal de determinar la frecuencia de los síntomas relacionados con los trastornos de la conducta disruptiva en pacientes con trastorno bipolar de inicio muy temprano, de inicio temprano y de inicio en el adulto, evaluados de manera retrospectiva. Nosotros evaluamos la hipótesis de que aquellos sujetos con TBP de inicio muy temprano mostraran mayor sintomatología externalizada comparada con aquellos que iniciaron el TBP a partir de los 13 años de edad.

\section{MATERIAL Y MÉTODOS}

\section{Muestra}

Todos los pacientes incluidos aceptaron participar de forma voluntaria mediante firma de consentimiento informado tras explicarles los objetivos de la investigación. Para los pacientes menores de edad, se solicitó de forma adicional el consentimiento informado de los padres o tutores legales.

Se incluyó a aquellos pacientes que cumplieran con los criterios diagnósticos del DSM-IV para el diagnóstico de trastorno bipolar I o trastorno bipolar tipo II, que a juicio del médico tratante se encontraran clínicamente estables y en condiciones de responder a la entrevista. Se excluyó a aquellos pacientes que tuvieran alguna enfermedad médica 
Trastornos externalizados y edad de inicio en pacientes con trastorno bipolar

concomitante que confundiera el diagnóstico de trastorno bipolar o si éste se presentaba de forma secundaria al uso de sustancias. Se eliminó del estudio a todos aquellos pacientes que no acudieron a dos citas y a aquellos que no completaron las evaluaciones clínicas programadas.

La muestra final estuvo conformada por 73 pacientes con trastorno bipolar I y II provenientes del Servicio de Consulta Externa ( $\mathrm{n}=25)$, la Clínica de Trastornos Afectivos $(n=38)$ y la Clínica de Adolescencia $(n=20)$ del Instituto Nacional de Psiquiatría Ramón de la Fuente Muñiz. El diagnóstico inicial se obtuvo mediante los criterios del DSM-IV y fue corroborado mediante la entrevista K-SADS-PL (siglas en inglés de Kiddie Schedule for Affective Disorders and Schizophrenia) versión México.

\section{INSTRUMENTOS Y PROCEDIMIENTO}

Para confirmar la presencia de un trastorno externalizado como el trastorno por déficit de atención con hiperactividad (TDAH), el trastorno de conducta (TC), el trastorno oposicionista desafiante (TOD), los pacientes fueron evaluados por medio del Kiddie-SADS-PL; para los pacientes adultos, las preguntas fueron adecuadas de forma retrospectiva. La K-SADS-PL es una entrevista diagnóstica semiestructurada diseñada para evaluar los episodios actuales y pasados de psicopatología en niños y adolescentes (6-18 años) de acuerdo con los criterios del DSM-III-R y DSM-IV, y que ha sido previamente validada en población mexicana. ${ }^{24}$ Para el resto de la comorbilidad psiquiátrica se empleó la MINI (Siglas en inglés de Mini International Neuropsychiatric Interview), en su versión original para adultos y la versión MINI Kid que se utiliza en niños y adolescentes. ${ }^{25}$ Una vez confirmados los diagnósticos y los criterios de inclusión y exclusión, se procedió a la aplicación de la Escala Autoaplicable de Evaluación de los Problemas Externalizados para Adolescentes y Adultos Jóvenes (EEPE-AA, anexo 2), instrumento conformado por cinco subescalas: a) inatención, b) oposicionistadesafiante, c) hiperactivo-impulsivo, d) disocial predatorio y e) disocial no predatorio. La EEPE-AA ha mostrado una adecuada validez y confiabilidad en población psiquiátrica mexicana. ${ }^{26}$

Las características demográficas (p. ej., edad, estado civil, escolaridad, ocupación) y clínicas (p. ej., estancia hospitalaria, edad de inicio) de los pacientes se obtuvieron mediante entrevista directa con el paciente y sus familiares. Esta información se recolectó en un formato previamente diseñado para el estudio.

A partir de la información obtenida, la muestra se dividió en tres grupos de acuerdo con la edad de inicio de los síntomas afectivos (ya sea inicio de los síntomas depresivos o maniacos/hipomaniacos): 1. Trastorno bipolar de inicio muy temprano (TBPIMT): Cuando el inicio del trastorno bipolar, ya sean los síntomas depresivos o los síntomas mania- cos/hipomaniacos, se presentó antes de los 13 años de edad. 2. Trastorno bipolar de inicio temprano (TBPIT): Cuando el inicio del trastorno bipolar, ya sean los síntomas depresivos o los síntomas maniacos/hipomaniacos, fue a partir de los 13 años hasta los 18 años de edad. 3. Trastorno bipolar de inicio en la vida adulta (TBIA): Cuando el inicio del trastorno bipolar, ya sean los síntomas depresivos o los síntomas maniacos/hipomaniacos, fue a partir de los 19 años de edad.

\section{Análisis estadístico}

Se utilizaron medidas de tendencia central y dispersión para las variables sociodemográficas. Además, para las variables categóricas se utilizaron $\chi^{2}$ de Pearson o prueba exacta de Fisher en el caso de que existieran menos de cinco mediciones por variable, y para variables dimensionales $\mathrm{T}$ deStudent para muestras independientes. Asimismo, se utilizaron ANOVAS simples para la comparación de los tres grupos determinados según la edad de inicio de los síntomas afectivos. Por último, se construyó un modelo interactivo de las variables en estudio por medio del software AMOS, obtenido mediante un análisis basado en el modelo de ecuaciones estructurales.

\section{RESULTADOS}

De la muestra inicial de 73 pacientes evaluados se descartó a ocho debido a que no completaron las evaluaciones iniciales correspondientes y a uno, porque su trastorno afectivo bipolar se relacionó con el uso de sustancias. De esta manera, la muestra final de nuestro estudio estuvo conformada por 64 pacientes.

En el grupo de inicio muy temprano (GIMT), la edad promedio del inicio del primer episodio depresivo fue tan temprana como a los 9.8 años (DE=1.9 años), comparado con la edad promedio en el grupo de inicio temprano (GIT) que fue de 16.5 años (DE=3.6 años) y con la del grupo de inicio en la adultez (GIA) que fue de 23.4 años de edad (DE=7.7 años). De acuerdo con esta clasificación, poco más del 70\% de los pacientes con TBP I y TBP II presentaron su primer episodio depresivo antes de los 18 años de edad. Se ubicó un mayor porcentaje de adolescentes en el (GIMT) en contraste con el porcentaje de adultos que reportaron su primer episodio depresivo antes de los trece años de edad. La edad de inicio de la disfunción fue menor en el GIMT en contraste con el GIT y el GIA. No se encontraron otras diferencias significativas en las variables demográficas restantes ni en la comorbilidad con trastornos externalizados de acuerdo con la edad de inicio del primer episodio depresivo (cuadro 1).

La edad de inicio de la disfunción (cuadro 2) fue significativamente menor en el GIMT (13.4 años, DE=3.3 años) comparada con el GIT (18.4 años, DE=4.9 años) y con el GIA (23.1 años, DE=6.6 años; F[2,59]=17.351,p<0.001). Aún cuan- 
Cuadro 1. Comparación de los porcentajes de variables clínicas y sociodemográficas a partir de la edad de inicio del primer episodio depresivo

\begin{tabular}{|c|c|c|c|c|c|c|c|}
\hline \multirow[b]{4}{*}{ Variable } & \multicolumn{6}{|c|}{ Primer episodio depresivo } & \multirow[b]{4}{*}{ Significancia } \\
\hline & \multicolumn{6}{|c|}{ Inicio } & \\
\hline & \multicolumn{2}{|c|}{ Muy temprano } & \multicolumn{2}{|c|}{ Temprano } & \multicolumn{2}{|c|}{ En el adulto } & \\
\hline & $\mathrm{n}$ & $\%$ & $\bar{N}$ & $\%$ & $\bar{N}$ & $\%$ & \\
\hline TBP tipo I & 16 & 32.0 & 21 & 42.0 & 13 & 26.0 & \multirow{2}{*}{$\chi^{2}(2)=0.955, p=0.620$} \\
\hline TBP tipo II & 6 & 46.2 & 4 & 30.8 & 3 & 23.1 & \\
\hline 18 años o menores & 10 & 55.6 & 8 & 44.4 & 0 & 0.0 & $\chi^{2}(2)=0.617, p=0.008$ \\
\hline 19 años o mayores & 12 & 26.7 & 17 & 37.8 & 16 & 35.6 & \multirow{2}{*}{$\chi^{2}(2)=0.079, p=0.961$} \\
\hline Trabajo remunerado & 10 & 33.3 & 12 & 40.0 & 8 & 26.7 & \\
\hline Femenino & 6 & 23.1 & 13 & 50.0 & 7 & 26.9 & \multirow{2}{*}{$\chi^{2}(2)=3.006, p=0.222$} \\
\hline Masculino & 16 & 43.2 & 12 & 32.4 & 9 & 24.3 & \\
\hline $\mathrm{TDAH}^{\mathrm{a}}$ & 6 & 46.2 & 4 & 30.8 & 3 & 23.1 & $\chi^{2}(2)=1.693, p=0.429$ \\
\hline Trastorno negativista desafiante ${ }^{a}$ & 1 & 33.3 & 1 & 33.3 & 1 & 33.3 & $\chi^{2}(2)=0.152, p=0.927$ \\
\hline Trastorno de conducta ${ }^{a}$ & 1 & 25.0 & 1 & 25.0 & 2 & 50.0 & $\chi^{2}(2)=1.047, p=0.592$ \\
\hline Trastornos externalizados ${ }^{a}$ & 6 & 42.9 & 4 & 28.6 & 4 & 28.6 & $\chi^{2}(2)=1.387, p=0.500$ \\
\hline
\end{tabular}

do no se reportaron diferencias significativas en las puntuaciones de las subescalas de la EEPE-AA, se observó una tendencia en el GIMT a presentar una mayor gravedad en las dimensiones evaluadas.

Primer episodio maniaco/hipomaniaco: No se encontraron diferencias en cuanto a las variables sociodemográficas del grupo (cuadro 3). La presencia del trastorno por déficit de atención con hiperactividad (TDAH), del trastorno negativista desafiante (TND), del trastorno de conducta (TC) y de la presencia de riesgo suicida al momento de la evaluación se relacionó con un inicio más temprano del TBP. No existieron diferencias significativas para el resto de los trastornos en los Eje I y II.

El promedio de edad al momento de la entrevista de los sujetos que pertenecieron al GIMT fue dos años mayor comparado con el GIT y éste a su vez fue significativamente menor que el GIA (cuadro 4). El promedio de edad del inicio del primer episodio hipomaniaco o maniaco fue significati- vamente menor en el GIMT (9.4 años, DE=2.2 años) comparado con el GIT (14.9 años, DE=1.4 años) y el GIA (24.3 años, $\mathrm{DE}=5.0 ; \mathrm{F}[2,61]=99.738, \mathrm{p}=0.001)$. La edad de inicio del primer episodio depresivo en el grupo de EIMT del primer episodio hipomaniaco/maniaco fue menor comparada con la edad de inicio del grupo de EIT. Los sujetos del GIMT reportaron una edad de inicio de la disfunción de 12.2 años con respecto al grupo de GIT y GIA de 16.5 y 23.9 años, respectivamente $(\mathrm{F}[2,60]=35.549, \mathrm{p}=0.0001)$.

En cuanto al tiempo promedio entre el inicio de la enfermedad y la búsqueda de atención, los sujetos del GIMT reportaron un intervalo promedio mayor comparados con el grupo de GIT y GIA (8.1 años, D.E.=6.9 años y 8.2 años, $\mathrm{DE}=6.2$ años, respectivamente $(\mathrm{F}[2,61]=3.201, \mathrm{p}=0.048)$. Con respecto a las puntuaciones obtenidas del EEPE-AA, se encontró que existieron diferencias significativas para todos los promedios comparados, obteniéndose en todas las subescalas mayores puntuaciones para el GIMT (cuadro 4).

Cuadro 2. Comparación de los promedios de variables clínicas y clinimétricas a partir de la edad de inicio del primer episodio depresivo

\begin{tabular}{|c|c|c|c|c|c|c|c|c|c|c|}
\hline \multirow[b]{4}{*}{ Variable } & \multicolumn{9}{|c|}{ Primer episodio depresivo } & \multirow[b]{4}{*}{ Significancia } \\
\hline & \multicolumn{9}{|c|}{ Inicio } & \\
\hline & \multicolumn{3}{|c|}{ Muy temprano } & \multicolumn{3}{|c|}{ Temprano } & \multicolumn{3}{|c|}{ En el adulto } & \\
\hline & $\bar{N}$ & $\bar{x}$ & $\mathrm{DE}$ & $\mathrm{N}$ & $\bar{x}$ & $\mathrm{DE}$ & $\mathrm{N}$ & $\overline{\mathrm{X}}$ & $\mathrm{DE}$ & \\
\hline Edad & 22 & 9.8 & 1.9 & 25 & 16.5 & 3.6 & 16 & 23.4 & 7.7 & $F(2,60)=5.827, p=0.005$ \\
\hline Edad de Inicio de la disfunción & 21 & 13.4 & 3.3 & 25 & 18.4 & 4.9 & 16 & 23.1 & 6.6 & $F(2,59)=17.351, p<0.001$ \\
\hline $\begin{array}{l}\text { Tiempo entre el inicio de la enfermedad } \\
\text { y la búsqueda de la atención }\end{array}$ & 22 & 12.4 & 8.8 & 25 & 7.8 & 5.9 & 16 & 7.7 & 7.0 & $F(2,60)=2.896, p=0.063$ \\
\hline EEPE AA* Inatención & 22 & 12.5 & 5.5 & 25 & 9.4 & 4.8 & 16 & 9.1 & 4.1 & $F(2,60)=3.135, p=0.051$ \\
\hline EEPE AA* Hiperactivo & 22 & 10.8 & 5.2 & 25 & 9.0 & 5.4 & 16 & 6.9 & 4.3 & $F(2,60)=2.653, p=0.079$ \\
\hline EEPE AA* Oposicionista desafiante & 22 & 4.0 & 2.5 & 25 & 3.4 & 2.7 & 16 & 2.3 & 2.0 & $F(2,60)=2.334, p=0.106$ \\
\hline EEPE AA* Disocial predatorio & 22 & 7.6 & 3.8 & 25 & 6.2 & 3.6 & 16 & 5.5 & 2.5 & $F(2,60)=1.989, p=0.146$ \\
\hline EEPE $A A^{*}$ Disocial no predatorio & 22 & 3.5 & 2.7 & 25 & 3.4 & 2.8 & 16 & 2.6 & 2.9 & $F(2,60)=0.631, p=0.536$ \\
\hline EPE AA* Total & 22 & 38.5 & 16.7 & 25 & 31.5 & 17.0 & 16 & 26.4 & 13.1 & $F(2,60)=2.749, p=0.072$ \\
\hline
\end{tabular}

*Escala de evaluación de problemas externalizados para adolescentes y adultos jóvenes. 
Cuadro 3. Comparación de los porcentajes de variables clínicas y sociodemográficas a partir de la edad de inicio del primer episodio maniaco

\begin{tabular}{|c|c|c|c|c|c|c|c|}
\hline \multirow[b]{4}{*}{ Variable } & \multicolumn{6}{|c|}{ Primer episodio maniaco } & \multirow[b]{4}{*}{ Significancia } \\
\hline & \multicolumn{6}{|c|}{ Inicio } & \\
\hline & \multicolumn{2}{|c|}{ Muy temprano } & \multicolumn{2}{|c|}{ Temprano } & \multicolumn{2}{|c|}{ En el adulto } & \\
\hline & $\mathrm{N}$ & $\%$ & $\mathrm{~N}$ & $\%$ & $\mathrm{n}$ & $\%$ & \\
\hline$\overline{\mathrm{TBP} I}$ & 10 & 19.6 & 25 & 49.0 & 16 & 31.4 & \multirow{2}{*}{$\chi^{2}(2)=2.842, p=0.241$} \\
\hline TBP ॥ & 4 & 30.8 & 3 & 23.1 & 6 & 46.2 & \\
\hline 18 años o menores & 6 & 33.3 & 12 & 66.7 & & & \multirow{2}{*}{$\chi^{2}(2)=12.241, p=0.002$} \\
\hline 19 años o mayores & 8 & 17.4 & 17 & 37.0 & 21 & 45.7 & \\
\hline Sin trabajo remunerado & 6 & 17.6 & 17 & 50.0 & 11 & 32.4 & \multirow{2}{*}{$\chi^{2}(2)=1.327, p=0.515$} \\
\hline Con trabajo remunerado & 8 & 26.7 & 11 & 36.7 & 11 & 36.7 & \\
\hline Femenino & 6 & 23.1 & 13 & 50.0 & 7 & 26.9 & \multirow{2}{*}{$\chi^{2}(2)=1.127, p=0.569$} \\
\hline Masculino & 8 & 21.1 & 15 & 39.5 & 15 & 39.5 & \\
\hline $\mathrm{TDAH}^{\mathrm{a}}$ & 7 & 53.8 & 4 & 30.8 & 2 & 15.4 & $\chi^{2}(2)=10.118, p=0.006$ \\
\hline Trastorno negativista desafiante ${ }^{a}$ & 3 & 100.0 & 0 & 0.0 & 0 & 0.0 & $\chi^{2}(2)=13.371, p=0.001$ \\
\hline Trastorno de conducta ${ }^{a}$ & 3 & 75.0 & 1 & 25.0 & 0 & 0.0 & $\chi^{2}(2)=7.163, p=0.028$ \\
\hline Trastornos externalizados ${ }^{a}$ & 7 & 50.0 & 5 & 35.7 & 2 & 14.3 & $\chi^{2}(2)=10.526, p=0.005$ \\
\hline
\end{tabular}

a Diagnóstico obtenido por K SADS PL.

La figura 1 muestra la comparación hecha entre la puntuación total promedio obtenida en cada grupo de edad de inicio, tanto para el episodio depresivo como para el episodio hipomaniaco/maniaco. Finalmente, por medio de un modelo de regresión lineal y un análisis de ecuaciones estructurales (figuras 1 y 2), encontramos que la edad al momento de la entrevista influyó en la obtención del diagnóstico de TDAH a lo largo de la vida. Los pacientes con diagnóstico de TDAH obtenido retrolectivamente presentan una mayor calificación en el EEPE-AA (anexo 1). Una menor edad de inicio del primer episodio de depresión se asoció con una menor edad de inicio del primer episodio hipomaniaco y/o maniaco $(\mathrm{p}<0.0001)$. Además, una menor calificación en el
EEPE-AA predijo una mayor edad de inicio en el primer episodio depresivo $(\mathrm{p}=0.013)$.

\section{DISCUSIÓN}

El abordaje en el estudio de la psicopatología a lo largo de la vida, tomando en cuenta la edad de inicio, propone un método de análisis que permite acercarse de manera más exacta a distintos riesgos de acuerdo con la aparición de la enfermedad. Aquí hay que resaltar que la identificación temprana y la intervención de las fases iniciales de cualquier trastorno mental de inicio en la infancia o en la adolescen-

Cuadro 4. Comparación de los promedios de variables clínicas y clinimétricas de acuerdo a la edad de inicio del primer episodio Hipomaniaco o Maniaco

\begin{tabular}{|c|c|c|c|c|c|c|c|c|c|c|}
\hline \multirow[b]{4}{*}{ Variable } & \multicolumn{9}{|c|}{ Primer episodio maniaco } & \multirow[b]{4}{*}{ Significancia } \\
\hline & \multicolumn{9}{|c|}{ Inicio } & \\
\hline & \multicolumn{3}{|c|}{ Muy temprano } & \multicolumn{3}{|c|}{ Temprano } & \multicolumn{3}{|c|}{ En el adulto } & \\
\hline & $\overline{\mathrm{N}}$ & $\bar{X}$ & $\mathrm{DE}$ & $\mathrm{N}$ & $\bar{x}$ & $\mathrm{DE}$ & $\mathrm{N}$ & $\bar{x}$ & $\mathrm{DE}$ & \\
\hline$\overline{\text { Edad }}$ & 14 & & 8.5 & 28 & 14.9 & 1.4 & 22 & 24.3 & 5.0 & $F(2,61)=99.7, p=0.0001$ \\
\hline Inicio del primer episodio depresivo & 14 & 10.9 & 4.7 & 28 & 14.0 & 3.0 & 21 & 23.4 & 5.3 & $F(2,60)=25.321, p=0.0001$ \\
\hline Edad de inicio de la disfunción & 14 & 12.2 & 4.7 & 28 & 16.5 & 3.6 & 21 & 23.9 & 4.6 & $F(2,60)=35.549, p=0.0001$ \\
\hline $\begin{array}{l}\text { Tiempo entre el inicio } \\
\text { de la enfermedad } \\
\text { y la búsqueda de la atención }\end{array}$ & 14 & 13.6 & 9.1 & 28 & 8.1 & 6.9 & 22 & 8.2 & 6.2 & $F(2,61)=3.201, p=0.048$ \\
\hline EEPE AA* Inatención & 14 & 13.5 & 5.8 & 28 & 11.0 & 4.2 & 22 & 7.3 & 4.2 & $F(2,61)=8.195, p=0.001$ \\
\hline EEPE AA* Hiperactivo & 14 & 12.1 & 5.4 & 28 & 10.2 & 4.9 & 22 & 5.3 & 3.5 & $F(2,61)=11.336, p=0.0001$ \\
\hline EEPE AA* Oposicionista desafiante & 14 & 5.2 & 2.6 & 28 & 3.6 & 2.4 & 22 & 1.7 & 1.4 & $F(2,61)=11.9, p=0.0001$ \\
\hline EEPE AA* Disocial predatorio & 14 & 8.6 & 4.0 & 28 & 7.0 & 2.8 & 22 & 4.2 & 2.9 & $F(2,61)=9.339, p=0.0001$ \\
\hline EEPE $A A^{*}$ Disocial no predatorio & 14 & 4.1 & 2.8 & 28 & 3.8 & 2.9 & 22 & 1.8 & 2.1 & $F(2,61)=4.947, p=0.01$ \\
\hline EEPE AA* Total & 14 & 43.6 & 17.6 & 28 & 35.7 & 14.3 & 22 & 20.3 & 11.7 & $F(2,61)=12.958, p=0.0001$ \\
\hline
\end{tabular}

*Escala de evaluación de problemas externalizados para adolescentes y adultos jóvenes. 


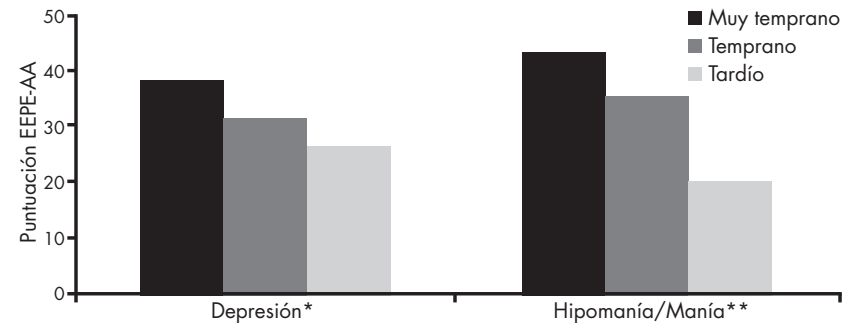

Figura 1. Comparación puntuaciones totales EEPE AA y los grupos por la edad inicio de los síntomas afectivos.

* Depresión, F $(1,62)=0.215, p=0.645 ;$ * * Hipomanía/Manía, F $(2,61)=$ $12.958, p=0.0001$.

cia, como lo es el TBP, podrían permitir modificar el curso clínico que muy probablemente sea tórpido y de difícil manejo. En general, el TBP de inicio muy temprano es una enfermedad con sustancial deterioro en dominios como el psicosocial y presenta un riesgo elevado para el suicidio, una significativa transmisión en familiares de primer grado y un curso prolongado del trastorno en el cual los ciclos clásicamente descritos, seguidos por periodos de bienestar, son observados rara vez.

El objetivo principal de este estudio fue evaluar en una muestra de adolescentes y adultos con TBP tipo I y II, que acuden a un hospital del tercer nivel de atención, la asociación de la edad de inicio, tanto para el primer episodio depresivo como para el primer episodio hipomaniaco/maniaco, con la presencia de síntomas relacionados con los trastornos externalizados (TDAH, TND Y TC). Con base en estos objetivos, nuestros datos confirman nuestra hipótesis principal, ya que aquellos sujetos que iniciaron su sintomatología antes de los 13 años mostraron una puntuación promedio mayor en el instrumento EEPE-AA comparado con el grupo de inicio en la adolescencia (entre los 13 y los 18 años) y con el grupo de inicio en la vida adulta (19 años o mayores).

El estudio de la relación entre el trastorno bipolar y los trastornos externalizados, en especial el trastorno por déficit de atención con hiperactividad, tiene un poco más de 20 años, desde los reportes de Biederman a finales del siglo pasado, ${ }^{8}$ donde mostró que el TDAH es un factor de riesgo para la presentación de un TBP con, además, un inicio más temprano. La asociación entre los trastornos externalizados, especialmente el TDAH y los trastornos afectivos en cuanto a su edad de inicio más temprano, tiene distintas implicaciones en cuanto a la salud, respuesta al tratamiento y un pronóstico más desfavorable.

La importancia del estudio de la edad de inicio y su asociación con los trastornos afectivos tipo bipolar radica en las implicaciones a nivel del curso y pronóstico de quien podría ser afectado por este trastorno. En nuestra muestra de pacientes, el tiempo entre el inicio de la enfermedad y la búsqueda de atención de manera general fue poco más de nueve años, sin haber encontrado diferencias por subtipo de trastorno bipolar. Una de las fortalezas y aportaciones

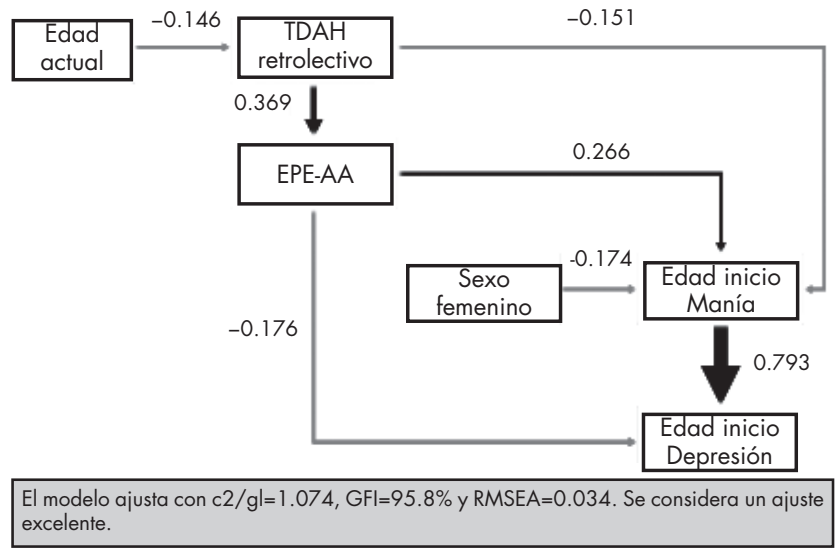

Figura 2. La edad de inicio del TBP y los síntomas de los trastornos externalizados: modelo predictivo (ver Anexo 1)

de nuestro estudio fue el análisis realizado desde la perspectiva del episodio depresivo y del episodio hipomaniaco/ maniaco. Para ambas perspectivas existieron dos datos que debemos destacar y que contribuyen a señalar que es necesario un cambio en la perspectiva sobre la conceptualización del TBP como un trastorno de inicio en la infancia y no de inicio en la adultez. El primero es que el inicio temprano de un episodio depresivo o de hipomanía/manía se asoció con un inicio más temprano del episodio complementario, ya sea hipomaniaco/manía o depresivo, respectivamente. El segundo dato, aún más preocupante, fue que para el grupo de inicio muy temprano, la edad de búsqueda de atención por este motivo de consulta fue, en promedio, de 12 años después del inicio de la sintomatología afectiva, seis años mayor comparado con los dos grupos restantes y dos años más si se evaluaba desde la perspectiva del primer episodio hipomaniaco/maniaco. Distintos estudios, como el reportado por Chong, ${ }^{27}$ muestran que la edad de inicio se asocia con la búsqueda de tratamiento, de tal manera que a menor edad de inicio existe una menor probabilidad de contacto para recibir tratamiento.

Dos razones que probablemente pudieron influir en este intervalo amplio entre la edad de inicio de la sintomatología y la búsqueda de atención, serían: 1. el carácter familiar de los trastornos afectivos en aquellos que inician a edades más tempranas, es decir que más de un familiar en primer grado estuviese afectado del mismo trastorno o del mismo espectro de trastornos, lo que a su vez puede contribuir a un umbral de reconocimiento más elevado, y 2. la no sospecha y por ende la no detección por los profesionales de la salud de primer nivel o contacto, lo que hace necesario mejorar los sistemas educativos y de salud donde se involucre a la sociedad civil, introduciendo objetivos de detección y tratamiento temprano en grupos de alto riesgo y de una visión de la psicopatología a lo largo de la vida, donde la mayor parte de ella se inicia en la infancia. Geller et al. ${ }^{28}$ han podido demostrar la importancia de la detección temprana y su implicación en el pronóstico, ya que aquellos sujetos 
con TBP de inicio en la infancia muestran, cuando alcanzan la adultez, características muy similares a aquellos adultos con TBP con un curso crónico, sin periodos de tiempo libres de sintomatología, de difícil manejo y con resistencia a los tratamientos psicofarmacológicos establecidos.

Otra de las razones para poder introducir la edad de inicio en la evaluación integral de nuestros pacientes es que ésta puede ser, para cualquier enfermedad psiquiátrica, un marcador de heterogeneidad etiológica y genética. ${ }^{29}$ Por esta razón el estudio de la edad de inicio ha sido útil para diferentes perspectivas, por ejemplo, la evaluación de fenotipos de un trastorno para asistir en el diagnóstico, en la determinación de la etiología o el pronóstico del trastorno en estudio $\mathrm{y}$, posiblemente, en la prevención y tratamiento del trastorno, en este caso, el trastorno bipolar. Estudiar un trastorno bipolar desde esta perspectiva implica relacionarlo con otros conceptos como el "efecto de cohorte o secular". $\mathrm{Al}$ respecto, algunos reportes epidemiológicos han indicado que los sujetos con trastorno bipolar que nacieron en 1940 o después tienen edades de inicio más tempranas comparados con aquellos que nacieron antes de 1940.30,31

La evaluación dimensional y sintomatológica de los trastornos externalizados en los sujetos con trastorno bipolar de nuestro estudio demostró una relación considerable entre éstos y el primer episodio hipomaniaco/maniaco. Se demostró con mayor fuerza la asociación de los síntomas relacionados con el trastorno por déficit de atención con hiperactividad, el trastorno oposicionista desafiante y el trastorno de conducta en aquellos sujetos con trastorno bipolar que iniciaron el primer episodio hipomaniaco/maniaco en la infancia. Este dato resalta la importancia del TDAH como un factor de mal pronóstico y de edad de inicio mucho más temprano de la psicopatología comórbida, en este caso, el trastorno bipolar.

Existen dos limitaciones principales de este estudio que deben ser mencionadas. Primero, no haber incluido el trastorno bipolar no especificado (TBP NE). Aunque el DSM-IV define de manera ambigua el TBP NOS, Birmaher et al ${ }^{32}$ definieron en el estudio COBY -uno de los estudios longitudinales más extensos e importantes en el área de investigación del trastorno bipolar pediátrico- con mayor precisión el TBP NE como la presencia de síntomas clínicamente relevantes de TBP que no reúnen completamente los criterios según el DSM-IV para TBP I y TBP II. En este estudio sólo el 8\% $(n=263)$ de la muestra presentaba un TBP II en el momento de la evaluación y el 35\% tenía un TBP NE. Los sujetos con TBP II, tuvieron un inicio de su trastorno afectivo más tardío y tuvieron índices más bajos de TDAH comórbido que los sujetos con TBP I y TBP NE ( $p<0=0.05)$. La importancia de considerar el TBP NE estriba en que muchos casos de inicio muy temprano o temprano se relacionan con este tipo de TBP y entonces pueden pasar desapercibidos, mostrando incluso un nivel comparable de disfunción con el TBP I. Segundo, no se pudo comprobar clinimétricamente que todos los pacientes estuvieran en eutimia, aunque los evaluadores al inicio de la entrevista tuvieron que comprobar clínicamente si los pacientes eran capaces de seguir la entrevista pues de lo contrario eran descartados.

Finalmente, tomando en cuenta las limitaciones y fortalezas ya comentadas, la implicación principal de nuestro estudio, la cual fue confirmada, es la de señalar la importancia de los trastornos externalizados, en especial del TDAH, como un predictor de una edad de inicio más temprana de otros trastornos mentales, en este caso, del trastorno bipolar.

\section{CONCLUSIONES}

El estudio del TBP desde la perspectiva de la edad de inicio ofrece una visión interesante que permite integrar elementos de la etapa del desarrollo que se está estudiando. Los trabajos realizados por distintos investigadores muestran que existe un curso único de aquellos sujetos con trastorno bipolar de inicio en la infancia, siendo más grave y crónico. La incorporación de los trastornos externalizados, especialmente el TDAH, como factores de riesgo, confirma la necesidad de tener una visión longitudinal y dinámica de la psicopatología a lo largo de la vida. Nuestro estudio, con sus limitaciones ya comentadas, pudo demostrar, desde una perspectiva transversal, que existen características clínicas, como la mayor presencia de trastornos externalizados asociados a una edad de inicio menor del trastorno bipolar.

\section{AGRADECIMIENTOS}

A las psicólogas Elena Mier y Terán y Raquel Soto por su participación en la evaluación clínica y por su invaluable apoyo.

A las doctoras Liz Sosa Mora, Claudia Becerra Palars y Doris Gutiérrez por su apoyo para que el equipo de evaluación pudiera estar en contacto con los pacientes en las Clínicas de Trastornos Afectivos y de la Adolescencia del INPRF.

A los pacientes y familiares por su participación entusiasta y desinteresada y sin quienes no habría sido posible la conclusión del presente estudio.

\section{REFERENCIAS}

1. Medina-Mora ME, Borges G, Benjet C, Lara C et al. Psychiatric disorders in Mexico: Lifetime prevalence in a nationally representative sample. British J Psychiatry 2007;190:521-528.

2. Merikangas KR, Jin R, He JP, Kessler RC et al. Prevalence and correlates of bipolar spectrum disorder in the world mental health survey initiative. Arch Gen Psychiatry 2011;68(3):241-251.

3. Charney DS. Bipolar disorder: can studies of natural history help us define clinically and neurobiologically relevant subtypes? Biol Psychiatry 2000;48:427.

4. Depp CA, Jin H, Mohamed S, Kaskow J et al. Bipolar disorder in middle-aged and elderly adults: is age of onset important? J Nerv Ment Dis 2004;92(11):796-799.

5. Shulman KI, Herrmann N. Bipolar disorder in old age. Can Fam Physician 1999;45:1229-1237. 
6. McGlashan T. Adolescent versus adult onset of mania. Am J Psychiatry 1988;145(2):221-223.

7. Rosen LN, Rosenthal NE, Van Dusen PH et al. Age at onset and number of psychotic symptoms in bipolar I and schizoaffective disorder. Am J Psychiatry 1983;140:1523-1524.

8. Ballenger JC, Reus VI, Post RM. The "atypical" clinical picture of adolescent mania. Am J Psychiatry 1982;139:602-606.

9. Rosenthal NE, Rosenthal LN, Stallone F et al. Toward the validation of RDC schizoaffective disorder. Arch Gen Psychiatry 1980;37:804-810.

10. Carter TD, Mundo E, Parikh SV, Kennedy JL. Early age at onser as a risk factor for por outcome of bipolar disorder. J Psychiatr Res 2003;37(4):297-303.

11. Carlson GA, Davenport YB, Jaminson K. A comparison of outcome in adolescent- and late-onset bipolar manic- depresive illness. Am J Psychiatry 1977;134:919-922.

12. Biederman J, Faraone S, Mick E, Wozniak J et al. Attention-deficit hyperactivity disorder and juvenile mania: an overlooked comorbidity? J Am Acad Child Adolesc Psychiatry 1996;35(8):997-1008.

13. Chang KD, Steiner $\mathrm{H}$ et al. Psychiatric phenomenology of child and adolescent bipolar offspring. J Am Acad Child Adolesc Psychiatry 2000;39:453-460.

14. Goldstein BI, Levitt AJ. Further evidence for a developmental subtype of bipolar disorder defined by age at onset: results from the national epidemiologic survey on alcohol and related conditions. Am J Psychiatry 2006;163(9):1633-1636.

15. Mick E, Biederman J, Faraone SV, Murray K et al. Defining a developmental subtype of bipolar disorder in a sample of nonreferred adults by age at onset. J Child Adolesc Psychopharmacol 2003;13(4):453-462.

16. Masi G, Mucci M, Pfanner C, Berloffa S et al. Developmental pathways for different subtypes of early-onset bipolarity in youths. J Clin Psychiatry 2012;73(10):1335-1341.

17. Perlis RH, Miyahara S, Marangell LB, Wisniewski SR et al. 24; STEPBD Investigators. Long-term implications of early onset in bipolar disorder: data from the first 1000 participants in the systematic treatment enhancement program for bipolar disorder (STEP-BD). Biol Psychiatry 2004;55(9):875-881.

18. Sachs GS, Baldassano CF et al. Comorbidity of attention deficit hyperactivity disorder with early- and late-onset bipolar disorder. Am J psychiatry 2000;157:466-468.

19. Kovacs M, Pollock M. Bipolar disorder and comorbid conduct disorder in childhood and adolescence. J Am Acad Child Adolesc Psychiatry 1995;34:715-723.

20. West $S$, Mc Elroy $S$ et al. Attention deficit hyperactivity disorder in adolescent mania. Am J Psychiatry 1995;152:271-274.
21. Wozniak J, Biederman J, Kiely K, Ablon JS et al. Mania-like symptoms suggestive of childhood onset bipolar disorder in clinically referred children. J Am Acad Child Adolesc Psychiatry 1995a;34:867-876.

22. Faraone SV, Biederman J et al. Attention- deficit hyperactivity disorder with bipolar disorder: a familial subtype? J Am Acad Child Adolesc Psychiatry 1997a;36:1378-1390.

23. Kessler RC, Adler L, Barkley R, Biederman J et al. The prevalence and correlates of adult ADHD in the United States: results from the National Comorbidity Survey Replication Am J Psychiatry 2006;163(4):716-723.

24. Ulloa RE, Ortiz S, Huiguera F, Nogales I et al. Estudio de fiabilidad interevaluador de la versión en español de la entrevista Schedule for Affective Disorders and Schizophrenia for School-Age Children-Present and Lifetime version (K-SADS-PL). Actas Esp Psiquiatr 2006;34(1):36-40.

25. Sheehan DV, Lecrubier Y, Harnett-Sheehan K, Janavs J et al. Reliability and validity of the Mini International Neuropsychiatric Interview (M.I.N.I), according to the SCID-P. European Psychiatry 1997;12:232-241.

26. Palacios L. Evaluación retrospectiva de la frecuencia de los síntomas relacionados a la conducta disruptiva en pacientes con trastorno bipolar de inicio muy temprano, de inicio temprano y de inicio en el adulto. Tesis para obtener el grado de Maestro en Ciencias Médicas, Odontológicas y de la Salud. México: Universidad Nacional Autónoma de México; 2010.

27. Chong SA, Abdin E, Sherbourne C, Vaingankar J et al. Treatment gap in common mental disorders: the Singapore perspective. Epidemiology Psychiatric Sciences 2012;21:195-202.

28. Geller B, Tillman R, Bolhofner K, Zimerman B. Child bipolar I disorder prospective continuity with adult bipolar I disorder; Characteristics of second and third episodes; Predictors of 8-year outcome. Arch Gen Psychiatry 2008;65(10):1125-1133.

29. Chengappa KNR, Kupfer DJ, Frank E, Houck PR et al. Relationship of birth cohort and early age at onset of illness in a bipolar disorder case registry. Am J Psychiatry 2003;160:1636-1642.

30. Gershon ES, Hamovit JH, Guroff JJ, Nurnberger JI. Birth-cohort changes in manic and depressive disorders in relatives of bipolar and schizoaffective patients. Arch Gen Psychiatry 1987;44:314-319.

31. Robins LN, Helzer JE, Weissman MM, Orvaschel $\mathrm{H}$ et al. Lifetime prevalence of specific psychiatric disorders in three sites. Arch Gen Psychiatry 1984;41:949-958.

32. Birmaher B, Axelson D, Strober M, Gill MK et al. Clinical course of children and adolescents with bipolar spectrum disorders. Arch Gen Psychiatry 2006;63(2):175-183.

Artículo sin conflicto de intereses 


\section{ANEXO 1 \\ Descripción del modelo interactivo de las variables en estudio}

\begin{tabular}{|c|c|c|c|c|c|}
\hline Variable dependiente & $\begin{array}{c}\text { Variable } \\
\text { independiente }\end{array}$ & $\begin{array}{l}\text { Beta } \\
\text { estándar }\end{array}$ & Z & $P$ & Observaciones \\
\hline Dx TDAH retrolectivo & Edad & -0.146 & 1.163 & 0.122 & $\begin{array}{l}\text { Los pacientes con menor edad tienen mayor } \\
\text { probabilidad de presentar el Dx de TDAH }\end{array}$ \\
\hline EPE-AA & $\begin{array}{l}\text { Dx TDAH } \\
\text { retrolectivo }\end{array}$ & 0.369 & 3.128 & 0.001 & $\begin{array}{l}\text { Los pacientes con Dx de TDAH presentan una } \\
\text { mayor calificación en EPE-AA }\end{array}$ \\
\hline Edad inicio hipomanía/manía & Sexo & -0.174 & 1.425 & 0.077 & $\begin{array}{l}\text { El sexo femenino se asocia a una menor edad } \\
\text { de inicio de la manía }\end{array}$ \\
\hline Edad inicio hipomanía/mania & $\begin{array}{l}\text { Dx TDAH } \\
\text { retrolectivo }\end{array}$ & -0.151 & 1.146 & 0.126 & $\begin{array}{l}\text { El Dx TDAH retrolectivo se asocia a una menor } \\
\text { edad de inicio de la manía }\end{array}$ \\
\hline Edad inicio hipomanía/manía & EEPE-AA & 0.206 & 1.565 & 0.059 & $\begin{array}{l}\text { Una mayor calificación en el EPE-AA se asocia } \\
\text { a mayor edad de inicio de la manía }\end{array}$ \\
\hline Edad inicio depresión & $\begin{array}{l}\text { Edad inicio } \\
\text { manía }\end{array}$ & 0.793 & 9.998 & 0.0001 & $\begin{array}{l}\text { Las edades de inicio de manía y depresión se } \\
\text { asocian de forma muy significativa }\end{array}$ \\
\hline Edad inicio depresión & EEPE-AA & -0.176 & 2.216 & 0.013 & $\begin{array}{l}\text { Una menor calificación en el EPE-AA se asocia } \\
\text { a mayor edad de inicio de la depresión }\end{array}$ \\
\hline
\end{tabular}




\section{ANEXO 2 \\ Escala de evaluación de problemas externalizados para adolescentes y adultos jóvenes (EEPE-AA)}

(Escala desarrollada en el Instituto Nacional de Psiquiatría RFM, México, 2002) Palacios Cruz L y cols.

Nombre:

Edad:

Expediente: Fecha:

Diagnóstico (llenado por el Médico):

\section{Instrucciones:}

Por favor lee con cuidado cada enunciado y marca con una " $\mathrm{X}$ " la respuesta que mejor represente la frecuencia con que has actuado. Es decir contesta qué tanto has actuado, en la forma que se te pregunta en cada enunciado a lo largo de toda tu vida para nunca, ocasionalmente o frecuentemente. Te aclaramos que no existen respuestas buenas - malas en este instrumento, además la información que se obtenga será conservada de manera confidencial por tu médico. Por favor, contesta toda la Escala.

\begin{tabular}{|c|c|c|c|c|}
\hline & & Nunca & $\begin{array}{c}\text { Ocasio- } \\
\text { nalmente }\end{array}$ & $\begin{array}{l}\text { Frecuen- } \\
\text { temente }\end{array}$ \\
\hline 1 & He tenido problemas para terminar las tareas escolares en la casa & & & \\
\hline 2 & He actuado como si tuviera un motor por dentro & & & \\
\hline 3 & He iniciado peleas físicas & & & \\
\hline 4 & He tenido problemas para concentrarme & & & \\
\hline 5 & He sido atento en los juegos & & & \\
\hline 6 & He discutido con los adultos & & & \\
\hline 7 & He cumplido las reglas en la casa y en la escuela & & & \\
\hline 8 & Te han dicho que parece que no escuchas cuando alguien te habla, es decir, no pusiste atención a lo que te decían & & & \\
\hline 9 & He tenido explosiones, pierdo el control & & & \\
\hline 10 & He sido organizado & & & \\
\hline 11 & Me he enojado por cosas insignificantes & & & \\
\hline 12 & He terminado las actividades que comienzo & & & \\
\hline 13 & Soy de los que habla mucho, mucho, mucho & & & \\
\hline 14 & Me ha molestado que me ordenen hacer cosas, me he rehusado a obedecer a pesar que tuvieran razón & & & \\
\hline 15 & He sido muy desesperado al esperar mi turno & & & \\
\hline 16 & He hecho berrinches & & & \\
\hline 17 & He tenido problemas para seguir instrucciones & & & \\
\hline 18 & Me he entrometido o interrumpido las conversaciones o juegos de otros & & & \\
\hline 19 & He sido alguien que no pone atención y/o olvida donde deja las cosas (ejemplo, llaves, carteras, libros) & & & \\
\hline 20 & Me ha costado trabajo mantenerme sentado (ejemplo, al comer o al estar en clases) & & & \\
\hline 21 & He sido muy escandaloso & & & \\
\hline 22 & He culpado a otros de mis errores de manera intencional, sabiendo que yo era el responsable de ellos & & & \\
\hline 23 & He molestado sólo por molestar & & & \\
\hline
\end{tabular}




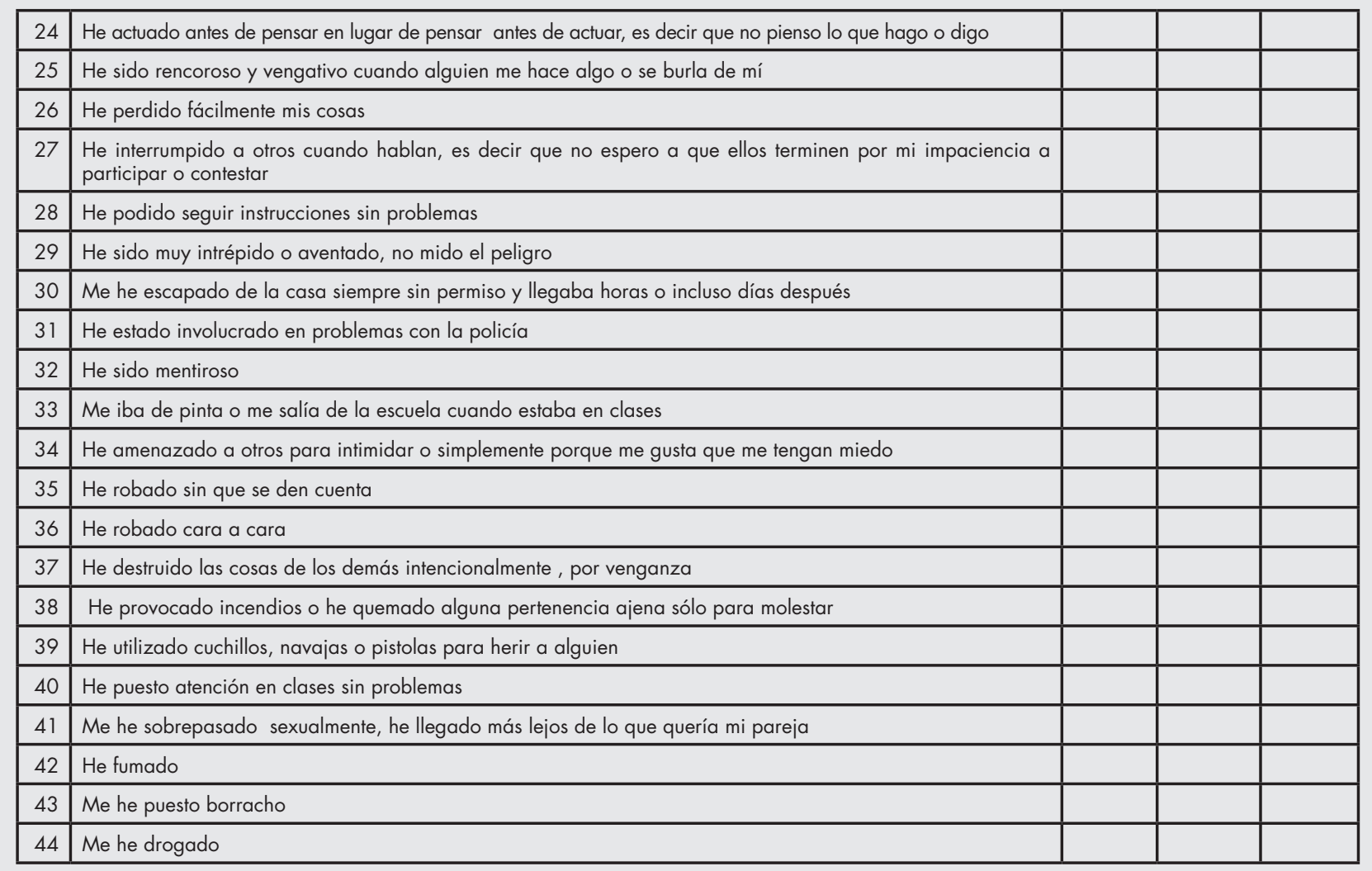

POR FAVOR REVISA HABER CONTESTADO TODOS LOS REACTIVOS 